\title{
Ebene und räumliche Rahmentragwerke
}

\author{
Von \\ Dr. Ing. Viktor Kupferschmid \\ Oberingenieur der Zentialverwaltung der \\ Bauunternehmung Carl Brandt, \\ Düsseldorf
}

Mit 252 Textabbildungen

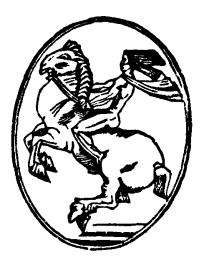

Wien

Springer-Verlag

1952 
ISBN-13: 978-3-7091-7804-1

e-ISBN-13: 978-3-7091-7803-4

DOI: 10.1007/978-3-7091-7803-4

Alle Rechte, insbesondere das der Übersetzung in fremde Sprachen, vorbehalten.

Copyright 1952 by Springer-Verlag in Vienna.

Softcover reprint of the hardcover 1st edition 1952 


\section{Vorwort}

Im vorliegenden Buch wird die Berechnung ebener und räumlicher Rahmentragwerke auf einheitlicher Grundlage behandelt. Es wendet sich in erster Linie an den jungen Fachkollegen.

Da durchgerechnete Beispiele am schnellsten und klarsten den Sinn einer Aufgabe vermitteln, wird auf sie ein besonderer Wert gelegt. Um die Beispiele jedoch nicht zu umfangreich zu gestalten, werden meist einfache, oft symmetrische Systeme behandelt. Die Rechengenauigkeit ist, um gute Übereinstimmung der Kontrollen zu erhalten, meist größer, als praktisch erforderlich wäre.

Einige Kapitel, wie z. B. die Berechnung von Rahmen mit Zugbändern, die Vereinfachungsmöglichkeiten bei der Berechnung räumlicher Rahmentragwerke durch Wahl bestimmter Stabquerschnitte, die Berechnung von durch Decken ausgesteifter räumlicher Rahmentragwerke und namentlich die im Anhang gebrachte unmittelbare Berechnung des zweiteiligen Stockwerksrahmens und des Virendeelträgers dürfte auch ältere Fachkollegen interessieren.

Sonst nicht übliche Bezeichnungen, wie z. B. „Grundverschiebungen“, „Grundgleichungen“ usw. sowie die Einteilung der räumlichen Rahmentragwerke sind nur gewählt, um eine klare, einheitliche Fassung zu erhalten. Auch der „Rahmen mit verschieblichen, aber unverdrehbaren Knoten" soll eine eindeutige Vorstellung vermitteln.

Die grundlegenden Gedanken der Behandlung der Rahmentragwerke in der vorliegenden Art folgen aus der „Deformationsmethode“ von Ostenfeld (Verlag Julius Springer, Berlin, 1925) und dem Momentenausgleichsverfahren von Cross. Das Buch entstand in den Jahren nach dem Kriege. Das Manuskript wurde im März 1949 abgeschlossen. Zur Zeit der Bearbeitung stand nur wenig Literatur zur Verfügung. Im Literaturverzeichnis sind jedoch auch einschlägige Werke mitaufgeführt, die zu einem späteren Zeitpunkt erschienen sind.

Den Herren Professoren Dr. Ing. E. Melan der Technischen Hochschule in Wien und Dr. Ing. Beer der Technischen Hochschule 
in Graz, zur Zeit an der Universität in Tucumán, Argentinien, möchte ich für ihr Interesse danken, das sie der Entstehung dieses Buches entgegengebracht haben.

Besonderen Dank schulde ich Herrn Otto Lange, Springer-Verlag. Der Springer-Verlag Wien hat die Herausgabe des Buches trotz der schwierigen Verhältnisse in der vorliegenden sorgfältigen Ausstattung ermöglicht.

Düsseldorf, im September 1951

V. Kupferschmid 


\title{
Inhaltsverzeichnis
}

\author{
Erster Teil
}

\section{Theoretische Grundlagen}

I. Allgemeines Verfahren zur Berechnung verschieblicher Rahmentragwerke . . . . . . . . . . . . . . . . 1

1. Festhaltungen bei Berücksichtigung und bei Vernachlässigung der Stablängskräfte in Rahmen mit geraden Stäben . . . . . 2

2. Festhaltungen bei Rahmen mit krummen Stäben oder Stabzügen . . . . . . . . . . . . . . . . . . . . . . . . . . . 3

3. Berechnung der Festhaltekräfte . . . . . . . . . . . . . . . 4

4. Belastung des verschieblichen Systems, Angriffskräfte. . . . . 4

5. Grundverschiebungen und Grundverschiebungszustände. . . . . 5

6. Kräftegruppen der Grundverschiebungen . . . . . . . . . . . 6

7. Ableitung der Grundgleichungen . . . . . . . . . . . . . . . 6

II. Das Momentenausgleichsverfahren von Cross. . . . . . . 9

1. Allgemeines . . . . . . . . . . . . . . . . . . . . . . . . 9

2. Erweiterte Fassung und Ableitung . . . . . . . . . . . . . . 9

3. Vorzeichenregel .. . . . . . . . . . . . . . . . . . . . . 13

4. Abklingungs- oder Übergangszahlen . . . . . . . . . . . . . . . . . . . . 14

5. Stabsteifigkeiten . . . . . . . . . . . . . . . . . . . . . . . . 15

6. Sonderfall: Rahmen mit geraden Stäben mit veränderlichen Trägheitsmomenten . . . . . . . . . . . . . 15

7. Sonderfall: Rahmen mit geraden Stäben mit konstanten Trägheitsmomenten . . . . . . . . . . . . . . . 16

8. Systemskizzen . . . . . . . . . . . . . . . . . . . . . . . 17

9. Zahlenbeispiel 1 . . . . . . . . . . . . . . . . . . . . . . . . . . 18

10. Allgemeine Vereinfachungen bei Rahmensymmetrie . . . . . . 23

11. Vereinfachungen bei symmetrischen Rahmen mit geraden Stäben mit konstanten Trägheitsmomenten . . . . . . . . . . . . . 24

12. Zahlenbeispiel 2 . . . . . . . . . . . . . . . . . . . . . . . 25

13. Allgemeinste Fassung des Crossschen Ausgleichsverfahrens . . . 27

III. Der Rahmen mit verschieblichen, aber unverdrehbaren Knoten . . . . . . . . . . . . . . . . . . . . 30

1. Grundlagen . . . . . . . . . . . . . . . . . . . . . . . . 30

2. Die Möglichkeiten zur Durchführung von Verschiebungen . . . 32

3. Kontrolle des Momentenausgleiches. . . . . . . . . . . . . . 33

4. Eigenarten des Rahmens mit verschieblichen, aber unverdrehbaren Knotenpunkten. . . . . . . . . . . . . . . . . . 33

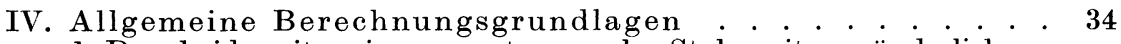

1. Der beiderseits eingespannte gerade Stab mit veränderlichem Trägheitsmoment . . . . . . . . . . . . . . . . . . . . . 2. Der einseitig eingespannte gerade Stab mit veränderlichem Trägheitsmoment 3

4

8

3

4

27

0

0


3. Der beiderseits eingespannte Bogen und Stabzug . . . . . . . 35

4. Parallelverschiebung der Stabenden beim beiderseits und einseitig eingespannten Stab . . . . . . . . . . . . . . . . . . . . 35

5. Die virtuelle Arbeit beim ebenen Rahmen . . . . . . . . . . 37

6. Formänderungswerte . . . . . . . . . . . . . . . . . . . . 41

7. Die virtuelle Arbeit bei schiefer (räumlicher) Stabbiegung . . . 43

Zweiter Teil

Ebene Rahmen

I. Übersicht . . . . . . . . . . . . . . . . . . 47

II. Rahmen mit geraden, aufeinander senkrecht stehenden

Stäben . . . . . . . . . . . . . . . . . . . . . . . 49

1. Zahlenbeispiel 3 . . . . . . . . . . . . . . . . . . . . . . . . . . . . . . 49

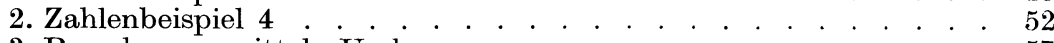

3. Berechnung mittels Umlagerung . . . . . . . . . . . . . . . 57

4. Zahlenbeispiel 5 . . . . . . . . . . . . . . . . . . . . . . 58

5. Zahlenbeispiel 6 . . . . . . . . . . . . . . . . . . . . . . . . . . . . . . . 59

III. Allgemeine Rahmenformen mit nur geraden Stäben . . . 61

1. Zahlenbeispiel 7 . . . . . . . . . . . . . . . . . . . . . . 62

2. Zahlenbeispiel 8 . . . . . . . . . . . . . . . . . . . . . . . . . . . . . . . . 66

3. Zahlenbeispiel 9 . . . . . . . . . . . . . . . . . . . . . . 74

IV. Rahmen mit Zugbändern . . . . . . . . . . . . . . . . . 78

1. Grundlagen . . . . . . . . . . . . . . . . . . . . . . . . 78

2. Zahlenbeispiel 10 . . . . . . . . . . . . . . . . . . . . 78

V. Rahmen mit krummen und polygonalen Stäben . . . . . 81

1. Der eingespannte Bogen und Stabzug . . . . . . . . . . . . 81

2. Das verschiebliche System . . . . . . . . . . . . . . . . . 84

3. Der einseitig gelenkig gelagerte Bogen oder Stabzug. . . . . . 85

4. Zahlenbeispiel 11 . . . . . . . . . . . . . . . . . . . . . . 87

5. Zahlenbeispiel 12 . . . . . . . . . . . . . . . . . . . . . . . . . . 88

6. Zahlenbeispiel 13 . . . . . . . . . . . . . . . . . . . . . . 88

VI. Rahmen mit nur aus zwei Stäben bestehenden Stabzügen 95

1. Grundlagen . . . . . . . . . . . . . . . . . . . . 95

2. Zahlenbeispiel 14 . . . . . . . . . . . . . . . . . . . . . . . . . . . 97

3. Zahlenbeispiel 15 . . . . . . . . . . . . . . . . . . . . . . 107

\section{Dritter Teil}

\section{Räumliche Rahmentragwerke}

I. Einteilung der räumlichen Rahmentragwerke . . . . . . 110

II. Räumliche Rahmentragwerke erster Ordnung . . . . . . 112

Zahlenbeispiel 16 . . . . . . . . . . . . . . . . . . . 112

III. Ausgleich von Biegungs- und Torsionsmomenten in räumlichen Rahmentragwerken erster Ordnung . . . . . . . . 121

1. Grundlagen . . . . . . . . . . . . . . . . . . 121

2. Der Einfluß der Torsionssteifigkeit auf das Berechnungsergebnis 125

3. Zahlenbeispiel 17 . . . . . . . . . . . . . . . . . . . 127 
IV. Umwandlung räumlicher Rahmentragwerke zweiter und dritter Ordnung im Rahmentragwerke erster Ordnung . 132

1. Grundlagen . . . . . . . . . . . . . . . . . . . . . . . . 132

2. Zahlenbeispiel 18 . . . . . . . . . . . . . . . . . . . . . . 140

V. Räumliche Rahmentragwerke zweiter Ordnung . . . . . 147

1. Räumlicher Momentenausgleich, Ausgleichsschema . . . . . . . 147

2. Beispiel für die Berechnung eines Ausgleichsschemas . . . . . 152

3. Zahlenbeispiel 19... . . . . . . . . . . . . . . . . . . . 154

4. Zahlenbeispiel 20 . . . . . . . . . . . . . . . . . . . . . . 165

VI. Durch Scheiben ausgesteifte räumliche Rahmentragwerke 171

1. Grundlagen . . . . . . . . . . . . . . . . . . . 171

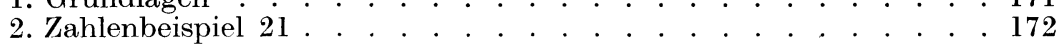

\section{Anhang}

\section{Unmittelbarer Ausgleich eines verschieblichen Systems}

I. Der symmetrische zweistielige Stockwerksrahmen . . . . 177

1. Grundlagen . . . . . . . . . . . . . . . . . . . . . . . . 177

2. Zahlenbeispiel 22 . . . . . . . . . . . . . . . . . . . . . . . . . 180

3. Zahlenbeispiel 23 . . . . . . . . . . . . . . . . . . . . . . . . . . . . 181

4. Zahlenbeispiel 24 . . . . . . . . . . . . . . . . . . . . . . 184

II. Der Virendeelträger . . . . . . . . . . . . . . . . . . . . 186

1. Grundlagen . . . . . . . . . . . . . . . . . . . . . . . . . 186

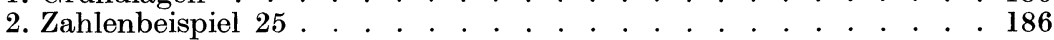

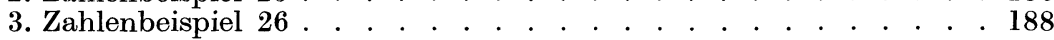

4. Zahlenbeispiel 27 . . . . . . . . . . . . . . . . . . . . . . 189

Literaturverzeichnis . . . . . . . . . . . . . . . . . . . . . 193

Sachverzeichnis . . . . . . . . . . . . . . . . . . . . . 195 